\title{
Museos en línea de Cataluña en la web 2.0 y en las redes sociales
}

Online museums of Catalonia in the Web 2.0 and social networks

\author{
Joan-Isidre BADELL \\ DigiDoc, Grupo de investigación, Universidad Pompeu Fabra, España \\ Doctorando Universidad de Barcelona, España \\ jibadell@gmail.com
}

\begin{abstract}
Resumen
Se analiza la presencia de los museos catalanes participantes en el proyecto Museos en línea en las redes sociales: si están, en cuáles de ellas, qué contenidos difunden, cómo se comunican con los usuarios, y la respuesta de éstos. La metodología utilizada se ha basado en dimensiones, parámetros e indicadores empleados en análisis semejantes por expertos cualificados adaptados al objetivo del estudio. Los resultados obtenidos muestran disparidad y diferencias significativas en relación con la importancia del museo, sus fondos y, previsiblemente, sus recursos económicos y humanos. Pero también demuestran la importancia de ser altamente activos y comunicar contenidos de calidad. Los datos más significativos, lamentablemente, se refieren a la escasa presencia en las redes sociales y a la casi nula implementación de herramientas web 2.0. En las conclusiones se aborda el necesario reto de mejorar en la implementación de las herramientas y en la presencia y actualización de la información que se difunde a través de las redes sociales.
\end{abstract}

Palabras clave: Museos. Cataluña. Redes sociales. Web 2.0. Comunicación cultural.

\section{Introdución}

Museos en línea tiene como objetivo poner a disposición del público la información que han ido generando los museos en su trabajo de documentación, conservación e investigación a lo largo de su existencia. Esta información, multiforme y heterogénea, que hasta hace bien poco no podía ser consultada si no era en el mismo museo, o en el mejor de los casos, en publicaciones impresas, ha visto ampliadas sus posibilidades de difusión con la presencia de internet.

Inicialmente, 42 museos catalanes (Anexo I), mayoritariamente de ciencias humanas, de un total de 107, han decidido participar en el proyecto voluntariamente. Según datos del propio proyecto, el resto de museos hasta completar los 107 se está preparando para su progresiva incorporación. En el momento de cierre del artículo (junio 2013) la web del proyecto anuncia

\begin{abstract}
The presence of the Catalan museums that participated in the Online Museum project in the social networks is analysed: whether they are present, in which ones, what contents they share, the way in which they communicate with the users, and their users' response. The methodology used was based on dimensions, parameters and indicators applied in similar analysis by qualified experts; and adapted to the specifities of this study. The results show disparity and significant differences among the museums, according to their importance, their funding and the expected financial and human resources. However, they also evidence the value of being highly active and communicate quality contents. Pitifully, the most significant results refer to their low presence in social networks and the almost null implementation of web 2.0 tools. The conclusions state the challenge of improving the implementation of adequate tools, the presence in social networks and the update of the information shared through them.
\end{abstract}

Keywords: Museums. Catalonia. Social networks. Web 2.0. Cultural communication.

la incorporación al mismo de nuevas instituciones hasta alcanzar los 80 museos participantes.

El proceso de transformación básicamente relacionado con el tratamiento unitario de la documentación de los museos catalanes se inició en 1982 (Museos en línea, s. a.)

[...] con la publicación del libro Sistema de documentación de museos que establecía las bases y sistematizaba todo el proceso de cómo documentar los objetos de un museo, homologando un libro de registro, unas fichas de inventario y un método de trabajo con la finalidad de conseguir un sistema unificado, útil a todos los museos de Cataluña y que posibilitara en un futuro el tratamiento informático de la información. Este paso se daba en 1992 con la creación del programa DAC (Documentación Asistida de Colecciones) fruto del acuerdo entre la Generalitat de Catalunya y el Ayuntamiento de Barcelona. Los cambios drásticos en las tecnologías de la información y el ritmo vertiginoso con que se produjeron llevaron a la adopción, en 2004, de un nuevo programa informático, MuseumPlus, 
que ha supuesto una notable mejoría en muchos aspectos, tanto en lo que respecta al funcionamiento de los propios museos como para los objetivos de documentación que el Departamento de Cultura de la Generalitat de Catalunya tiene marcados por la Ley de Museos de 1990.

La primera versión de Museos en línea entró en funcionamiento en 2008. Después de las necesarias correcciones tras un período de prueba y de evaluación de las funcionalidades del sistema, desde noviembre de 2009 se utiliza la segunda versión del programa, que posibilita el acceso a una parte importante de la documentación de los museos catalanes".

El reto final al cual el programa Museos en línea se enfrenta ahora es el de una "apertura del programa a la aportación de los usuarios y al intercambio de datos entre instituciones y entre investigadores." Dicho de otra manera, estar presente e interactuar en las redes sociales.

Este reto del proyecto es el que se ha tomado como referente para el estudio que se presenta a continuación. El objetivo es presentar el resultado de un análisis cuantitativo y cualitativo de las sedes web de los 42 museos (Anexo I) participantes en el proyecto. No se analiza el contenido museístico, las colecciones ni la herramienta de consulta del proyecto; sino que se centra en la presencia en las redes sociales de las instituciones participantes y en el uso de herramientas básicas de comunicación 2.0, es decir, en el estado de situación a día de hoy en estos aspectos. Tomando como referencia los estudios de Arends (2011), Arroyo-Vázquez (2009), Burton (2012), Celaya (2009), Kelly (2010), García Marco (2008) y Quijano (2012) sobre la necesidad de promoción, interacción y difusión de los museos en las redes sociales; y, más específicamente, el estudio de Gómez Vílchez (2012) -dedicado a los museos españoles exclusivamente-, centrado en el proceso de evaluación participativa entre museos y redes sociales.

La literatura profesional es muy amplia en estudios sobre museos, redes sociales y aplicaciones tecnológicas. De hecho, existen dos congresos internacionales dedicados a ello: $\mathrm{Mu}$ seum and the web, que se celebra anualmente en Estados Unidos o Canadá; y Museum Next, el equivalente del anterior a nivel europeo , quecelebró su primera edición en Barcelona el 2012. En España, el Museo de Arte Contemporáneo de Castilla y León (MUSAC) ha celebrado dos jornadas de trabajo sobre el tema en 2011 y 2012; y la asociación española DosDoce.com ha elaborado interesantísimos estudios (2006, 2009 y 2013) sobre la situación de los museos con las redes sociales y las últimas herramientas tecnológicas en nuestro país.
La pregunta de investigación planteada es la siguiente: ¿los museos participantes en el proyecto Museos en línea están utilizando las herramientas básicas de comunicación de última generación y están presentes informando, actualizando e interactuando con los usuarios a través de las redes sociales? Se parte de la premisa que estos museos, por el mero hecho de participar en un proyecto colaborativo de difusión de sus fondos en internet, estarán predispuestos a estar presentes en las redes sociales y a la implementación en sus webs de herramientas de comunicación de última generación.

\section{Metodología}

Se ha tomado la lista de museos participantes para su análisis de la sede web del proyecto. El análisis se ha realizado durante el mes de febrero de 2013. El primer paso ha sido verificar la dirección web que consta en el directorio del proyecto, disponible desde la página web del Departamento de Cultura de la Generalitat de Catalunya, con la real del museo. Se constató que no constaba la url en algunos museos, tres en concreto, en el directorio; y que otros tres museos no tenían la url actualizada y daban error en la conexión.

Para la realización del análisis se ha definido la necesidad de obtención de datos de tipo cuantitativo y de tipo cualitativo aplicado a los cibermedios según las indicaciones de RodríguezMartínez, Codina y Pedraza (2012) y Monistrol, Codina y Rovira (2006), adaptándolas al escenario de los museos, caso de nuestro estudio.

El escenario en el que se despliega la metodología es el contenido de los sitios web de los museos, con especial atención a los recursos digitales que éstos han desarrollado o aplicado para hacerlos llegar de forma adecuado a sus usuarios.

Los indicadores adaptados seleccionados han sido agrupados en dimensiones y parámetros.

Las dimensiones, consideradas partes mesurables de un cuerpo, en nuestro estudio, la sede web, nos facilitan la proporción o la forma de la misma para su análisis. Dicho de otro modo, son la expresión de una magnitud en relación a una serie de parámetros asignados. Se han estudiado seis dimensiones: cooperación, participación, creación de contenidos, acceso al contenido, socialización y comunicación.

En lo que se refiere a los parámetros analizados, se han evaluado seis -que nos permiten valorar, especialmente, la adaptación del museo a la interacción de la web 2.0-: interacción usuario-institución, publicación de contenidos, 
registro de usuarios y seguidores, acceso a la información, empleo de herramientas web 2.0, y plataformas de la web 2.0 en las que tiene presencia la institución.

En lo que respecta a los indicadores se han seleccionado los cuatro siguientes:

- Creación y mantenimiento de blogs, publicación de textos, publicación de fotos y/o vídeos.

- Registro por parte del usuario, contacto con otros usuarios, y los "Me gusta".

- Acceso a la información en portada, secciones, noticias, buscador, web, mapa web, recomendación de otros usuarios, subscripción de alertas, boletines y RSS.

- Presencia en servicios o plataformas de redes sociales, fecha de alta de la institución, actualización de la información, presentación, finalidad de los contenidos, y público de destino.

Además se ha analizado la sede web de los museos para comprobar la adaptación y utilización de herramientas de interacción, como la posibilidad de visitar el museo a través de un recorrido virtual (visita o tour virtual), la subscripción a servicios sindicados de noticias (RSS) desde la web y el contacto con la institución y responsable de la web, en la misma línea de otra investigación previa realizada anteriormente con otros casos (Badell, 2011; Badell, Rovira, 2010).

\section{Resultados}

Se ha realizado un análisis de los museos participantes en el proyecto comprobando si están registrados oficialmente en servicios de redes sociales. De los 42 museos participantes, 24, (Gráfico I y Tabla I) están presentes en alguna plataforma. Poco más de la mitad (57\%), no parece un resultado muy alto a tenor de uno de los objetivos del proyecto, aunque se defina como objetivo de futuro.

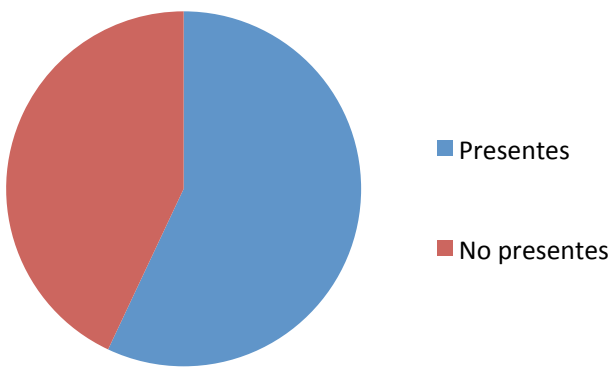

Gráfico I. Presencia en las redes sociales

\begin{tabular}{|c|c|}
\hline Museo & Tipología de redes sociales \\
\hline $\begin{array}{l}\text { Biblioteca Museo Víctor Balaguer. } \\
\text { Vilanova i la Geltrú (Barcelona) }\end{array}$ & $\begin{array}{l}\text { Flickr, Facebook, Blog (des 1- } \\
\text { 12-2011) }\end{array}$ \\
\hline $\begin{array}{l}\text { Museo de Arte Jaume Morera. } \\
\text { Lleida }\end{array}$ & $\begin{array}{l}\text { Flickr, Facebook, YouTube, } \\
\text { Twitter, Pinterest, Foursquare, } \\
\text { Google+, }\end{array}$ \\
\hline $\begin{array}{l}\text { Museo de Arte Moderno de } \\
\text { Tarragona }\end{array}$ & $\begin{array}{l}\text { Flickr, Facebook, YouTube, } \\
\text { Twitter, }\end{array}$ \\
\hline $\begin{array}{l}\text { Museo de Historia Sant Feliu de } \\
\text { Guíxols (Girona) }\end{array}$ & Facebook, Twitter, Google+ \\
\hline $\begin{array}{l}\text { Museo de Badalona. Badalona } \\
\text { (Barcelona) }\end{array}$ & Facebook, YouTube \\
\hline $\begin{array}{l}\text { Thermalia. Museo de Caldes de } \\
\text { Montbui (Barcelona) }\end{array}$ & Facebook \\
\hline $\begin{array}{l}\text { Museo Comarcal de Cervera } \\
\text { (Lleida) }\end{array}$ & Facebook \\
\hline Museo de Gavà (Barcelona) & Facebook- Twitter, YouTube \\
\hline $\begin{array}{l}\text { Museo de l'Empordà. Figueres } \\
\text { (Girona) }\end{array}$ & Facebook \\
\hline $\begin{array}{l}\text { Museo de Montserrat. Monistrol de } \\
\text { Montserrat (Barcelona) }\end{array}$ & Facebook, Twitter, Blog \\
\hline Museo del Cine. Girona & $\begin{array}{l}\text { Facebook, Twitter, YouTube, } \\
\text { Linkdln }\end{array}$ \\
\hline $\begin{array}{l}\text { Museo del Juguete de Catalunya. } \\
\text { Figueres (Girona) }\end{array}$ & Facebook \\
\hline $\begin{array}{l}\text { Museo de la Ciencia y la Técnica } \\
\text { de Catalunya. Terrassa } \\
\text { (Barcelona) }\end{array}$ & $\begin{array}{l}\text { Facebook, Twitter, Youtube, } \\
\text { Linkdln }\end{array}$ \\
\hline $\begin{array}{l}\text { Museo Episcopal de Vic } \\
\text { (Barcelona) }\end{array}$ & $\begin{array}{l}\text { Flickr, Facebook, Twitter, } \\
\text { Delicious, Vimeo }\end{array}$ \\
\hline $\begin{array}{l}\text { Can Quintana. Museo de la } \\
\text { Mediterránea. Torroella de Montgrí } \\
\text { (Girona) }\end{array}$ & Facebook, Twitter \\
\hline $\begin{array}{l}\text { Museo Marítimo de Barcelona. } \\
\text { Barcelona }\end{array}$ & $\begin{array}{l}\text { Flickr, Facebook, YouTube, } \\
\text { Twitter, Linkdln, Blogs }\end{array}$ \\
\hline $\begin{array}{l}\text { Museo Molí Paperer de } \\
\text { Capellades (Barcelona) }\end{array}$ & Facebook, Twitter \\
\hline $\begin{array}{l}\text { Museo Industrial del Ter. Manlleu } \\
\text { (Barcelona) }\end{array}$ & Facebook, Twitter, YouTube \\
\hline $\begin{array}{l}\text { Museo Nacional de Arte de } \\
\text { Catalunya. Barcelona }\end{array}$ & $\begin{array}{l}\text { Flickr, Facebook, Twitter, } \\
\text { Vimeo }\end{array}$ \\
\hline $\begin{array}{l}\text { Ecomuseo de les Valls d'Àneu } \\
\text { (Lleida) }\end{array}$ & Facebook \\
\hline $\begin{array}{l}\text { Museo de la Vida Rural. L'Espluga } \\
\text { de Francolí (Tarragona) }\end{array}$ & $\begin{array}{l}\text { Flickr, Facebook, Twitter, } \\
\text { YouTube, issuu, Blog }\end{array}$ \\
\hline $\begin{array}{l}\text { Vinseum. Museo de las Culturas } \\
\text { del Vino de Catalunya. Vilafranca } \\
\text { del Penedès (Barcelona) }\end{array}$ & Facebook, Twitter, YouTube \\
\hline $\begin{array}{l}\text { Museo Romántico Can Papiol. } \\
\text { Vilanova i la Geltrú (Barcelona) }\end{array}$ & Facebook \\
\hline $\begin{array}{l}\text { Museo del Ferrocarril. Vilanova i la } \\
\text { Geltrú (Barcelona) }\end{array}$ & $\begin{array}{l}\text { Flickr, Facebook, Twitter, } \\
\text { YouTube, Issuu }\end{array}$ \\
\hline
\end{tabular}

Tabla I. Museos presentes en redes sociales

Los servicios de redes sociales presentan una clara diversificación, que permite agruparlos en dos bloques bien diferenciados. El primero incluye a los servicios más populares o utilizados (Gráfico II): Facebook, que con 24 museos (todos los que tienen alguna cuenta) representa el $57 \%$; Twitter, con 16 museos, un $38 \%$; YouTube 
lo usan 11 museos, un 26\%; y finalmente Flickr solo 8 , lo que representa el $19 \%$. El otro bloque (Otros en el gráfico) viene representado por espacios colaborativos menos conocidos. Así, Issuu y Vimeo aparecen en dos museos, y Delicious, Pinterest y Foursquare solo contabilizan uno. Exclusivamente tres museos están presentes en Linkedln, la red entre profesionales más utilizada a nivel mundial.

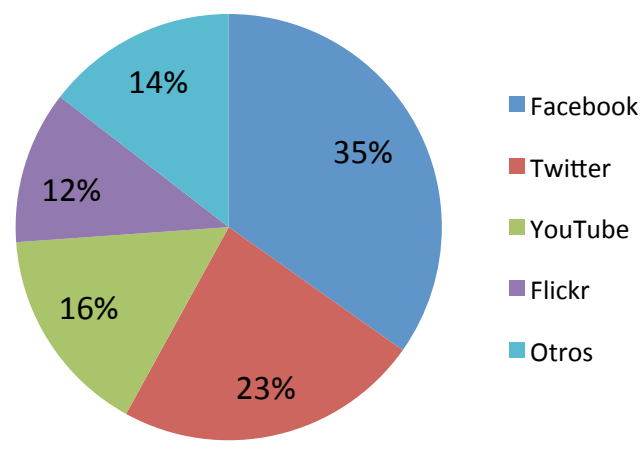

Gráfico II. Redes sociales

Es destacable, de forma negativa, que $7 \mathrm{mu}-$ seos no disponen de web propia, sino que dependen de la de su institución, mayoritariamente del Ayuntamiento correspondiente. La información facilitada suele ser meramente informativa: dirección, contacto, acceso, horarios, información general, fondos o colecciones más importantes y poca cosa más. Por tanto, son 35 los museos que disponen de una web propia.

Resumiendo este apartado, el $57 \%$ de los museos participantes en Museos en línea están presentes de alguna manera en las redes sociales y un $43 \%$ no lo están de ninguna de las maneras, es decir, están completamente ausentes (Anexo II). Sólo 11 museos (26\%) tienen subscripción sindicada de noticias RSS, y todavía muchos menos, sólo 3 museos $(7 \%)$-aunque 5 lo indican - disponen de visita o tour virtual en su web; los otros 2 tienen disponible videos de presentación.

De los 4 museos que mantienen un blog (Tabla II), hay dos que mantienen un buen nivel de actualización con comentarios, noticias, actividades, etc., que son la Biblioteca Museo Víctor Balaguer y el Museo Marítimo. Los otros dos son el Museo de la Vida Rural —que se registró en 2009, pero ha ido perdiendo actualización hasta el 2012 en que hay pocas entradas-, y el Museo de Montserrat —que, teniendo el blog bajo la responsabilidad del director, apenas se actualiza-. Hay un quinto museo que mantiene un blog, el Diocesano de Tarragona, que no es propio del museo, sino que pertenece al Arzo- bispado; y por eso no se ha tenido en cuenta en el análisis. El museo más activo y participativo es el Marítimo de Barcelona, que mantiene 4 blogs diferenciados: Actividades, Bitácoras, Educación, y FotoMar. El segundo, Bitácoras es el más activo, además de ser el más antiguo (2 de octubre de 2008). Ya cuenta con 13 entradas este 2013 (hasta mediados de febrero); y publicó 47 en el 2012 y 57 en el 2009, por proporcionar una perspectiva histórica. La participación de los ciudadanos es escasa en general en todos ellos, los más activos son los usuarios del Marítimo. Este dato plantea un par de preguntas: ¿el esfuerzo que conlleva mantener un blog merece la pena ante tan poca participación de los usuarios? ¿Utilizan el blog los usuarios como un mero servicio de información más y no lo ven como un canal de participación, opinión y de interactuar? Cuestiones que Simon (2007) ya planteaba en su obra y que no parecen todavía haberse resuelto.

\begin{tabular}{ll}
\hline Museo & Características \\
\hline Biblioteca & Fecha de alta: 1/12/2011. Buena actualización. \\
Museo Víctor & Novedades, noticias sobre actividades, \\
Balaguer. & comentarios. Incluyen fotos de calidad. Bien \\
Vilanova i la & redactado, ameno. Nula participación de \\
Geltrú & usuarios, sin comentarios en la mayoría de \\
(Barcelona) & entradas. \\
\hline Museo de & Fecha de alta: 1/11/2009. Realizado por el \\
Montserrat. & director del museo. No está actualizado, \\
Monistrol de & consultado el 12/02/13, última entrada del 16 de \\
Montserrat & enero. Solo dos entradas en todo el año 2012 \\
(Barcelona) & comparado con las 11 del 2011 y las 10 del 2010. \\
& Entradas muy largas y densas. Incluyen fotos e \\
& ilustraciones. Pocos comentarios (uno o dos a lo \\
sumo) en algunas entradas.
\end{tabular}

Tabla II. Museos que mantienen un blog

En Flickr (Tabla III) tienen cuenta un total de 8 museos, datos que se antojan francamente bajos. Flickr es idóneo para colgar los reportajes fotográficos, álbumes de fotos para compartir, opinar sobre ellas o para descargarlas. Ideal para mostrar gráficamente el edificio, las salas, la colección y las actividades realizadas. 
En Youtube (Tabla IV), servicio de red social caracterizado por el intercambio de videos, tienen cuenta 11 museos del proyecto. El primer museo en tener cuenta en Youtube fue el Museo de la Ciencia y la Técnica de Terrassa en noviembre de 2007. Durante el año siguiente, 2008, se incorporaron el Museo de Badalona, el Museo Industrial del Ter y el Museo de la Vida Rural. Los once museos cumplen de sobras con la función específica de este espacio, subir y mantener al día los videos y video-reportajes de las actividades y acontecimientos del museo. En cuanto al número de visualizaciones como podemos observar es muy divergente según las características de cada centro. Se observa que los acontecimientos locales o de interés local son los más vistos, como ejemplo, el video de la llegada de la locomotora Talgo en el Museo del Ferrocarril o, la cata de vinos en el Museo del Vino.

\begin{tabular}{ll}
\hline Museo & Características \\
\hline Biblioteca Museo Víctor Balaguer. Vilanova i la Geltrú (Barcelona) & Galería fotográfica actualizada desde marzo de 2011 \\
\hline Museo de Arte Jaume Morera. Lleida & Galería fotográfica y videográfica actualizada desde enero de 2010 \\
\hline Museo de Arte Moderno de Tarragona & Galería fotográfica y videográfica actualizada desde julio de 2010 \\
\hline Museo del Ferrocarril. Vilanova i la Geltrú (Barcelona) & Galería fotográfica desde mayo de 2011 \\
\hline Museo Episcopal de Vic (Barcelona) & Galería fotográfica desde mayo de 2009 \\
\hline Museo Marítimo de Barcelona & Galería fotográfica desde febrero de 2010 \\
\hline Museo Nacional de Arte de Catalunya. Barcelona & Numerosa galería fotográfica desde enero de 2012 \\
\hline
\end{tabular}

Tabla III. Flickr

\begin{tabular}{|c|c|c|c|c|}
\hline Museo & Creación & Repr. & Videos & Más reproducido \\
\hline Museo de Arte Jaume Morera. Lleida & $27-11-2009$ & 2.612 & 20 & $\begin{array}{l}\text { Documental sobre el Museo del Agua de } \\
\text { Lleida (3.495) }\end{array}$ \\
\hline Museo de Arte Moderno de Tarragona & 03/10/2010 & 851 & 10 & La obra “La Comella” Rufino Mesa (303) \\
\hline Museo de Badalona. Badalona (Barcelona) & $04 / 03 / 2008$ & 1.186 & 5 & Inauguración del museo (480) \\
\hline Museo de Gavà (Barcelona) & $25 / 05 / 2011$ & 1.176 & 8 & $\begin{array}{l}\text { Sobre el Museo de Arqueología de } \\
\text { Catalunya (348) }\end{array}$ \\
\hline Museo del Cine. Girona & 09/11/2009 & 150.652 & 94 & Varios superan las 6.000 visualizaciones \\
\hline $\begin{array}{l}\text { Museo de la Ciencia y la Técnica de Catalunya. Terrassa } \\
\text { (Barcelona) }\end{array}$ & $01 / 11 / 2007$ & 622.360 & 30 & $\begin{array}{l}\text { Sobre el Museo de la Minas de Bellmunt } \\
\qquad(7.089)\end{array}$ \\
\hline Museo Industrial del Ter. Manlleu (Barcelona) & $25 / 03 / 2008$ & 4.825 & 38 & Sobre la oferta educativa del museo (800) \\
\hline Museo del Ferrocarril. Vilanova i la Geltrú (Barcelona) & $11 / 05 / 2011$ & 3.008 & 17 & $\begin{array}{l}\text { Sobre la llegada de la locomotora Talgo } \\
\qquad(974)\end{array}$ \\
\hline Museo Marítimo de Barcelona. & $19 / 04 / 2011$ & 236 & $2[46]$ & De Travesía con Sta. Eulalia (165) \\
\hline $\begin{array}{l}\text { Vinseum. Museo de las Culturas del Vino de Catalunya. Vilafranca } \\
\text { del Penedès (Barcelona) }\end{array}$ & $14 / 03 / 2012$ & 2 & $1[7]$ & Taste (I) (237) \\
\hline Museo de la Vida Rural. L'Espluga de Francolí (Tarragona) & $27 / 05 / 2008$ & 9.497 & 24 & $\begin{array}{l}\text { Joan Ponç - JV Foix, la obra “La pell de la } \\
\text { pell (1.537) }\end{array}$ \\
\hline
\end{tabular}

Tabla IV. Youtube

El servicio de Twitter (Tabla V) es, tal vez, el que en estos momentos goza de mayor uso entre la ciudadanía, por lo que resulta todavía más abrumadora la cifra tan baja de museos registrados, solo 16 . Es realmente preocupante esta masiva ausencia ante la realidad de difusión y participación de Twitter en todos los ámbitos. En líneas generales, el nivel de participación es alto y la actualización al día. No obstante, se ha detectado que algunos museos no ofrecen los datos públicamente. Alguno tiene una actualización discontinua; e incluso uno ha dado error en el enlace. El museo con mayor número de seguidores es el Museo Nacional de Arte de Catalunya con más de 15.000 , seguido del Museo de la Vida Rural —una sorpresa, ya que se trata de un museo local, muy activo por cierto en todas las redes sociales, pues, como se observa en la tabla $\mathrm{V}$, es el que más publica- y a continuación el Marítimo de Barcelona con casi 2.000 seguidores y el de la Ciencia y la Técnica de Terrassa con cerca de los 1.500. 


\begin{tabular}{|c|c|c|c|c|}
\hline Museo & Tuits & Siguiendo & Seguidores & Último publicado \\
\hline Museo de Arte Jaume Morera. Lleida & 383 & 930 & 1.108 & $09 / 02 / 13$ \\
\hline Museo de Arte Moderno de Tarragona & \multicolumn{4}{|c|}{ Error en el enlace "Disculpa. No existe" } \\
\hline Museu de Historia de Sant Feliu de Guíxols (Girona) & \multicolumn{4}{|c|}{ No actualizado. Sin datos. Último publicado: julio 2012} \\
\hline Museo de Gavà (Barcelona) & 438 & 255 & 503 & $08 / 02 / 13$ \\
\hline Museo de Montserrat. Monistrol de Montserrat (Barcelona) & 847 & 356 & 704 & $13 / 02 / 13$ \\
\hline Museo del Cine. Girona & 1.391 & 1.813 & 2.187 & $13 / 02 / 13$ \\
\hline Museo de la Ciencia y la Técnica de Catalunya. Terrassa (Barcelona) & 1.620 & 609 & 1.451 & $13 / 02 / 13$ \\
\hline Museo Episcopal de Vic (Barcelona) & \multicolumn{4}{|c|}{ Actualizado, pero sin datos } \\
\hline Can Quintana. Museo de la Mediterránea. Torroella de Montgrí (Girona) & 1.594 & 707 & 1.100 & $13 / 02 / 13$ \\
\hline Museo Marítimo de Barcelona & 996 & 1.086 & 1.931 & $13 / 02 / 13$ \\
\hline Museo Molí Paperer de Capellades (Barcelona) & \multicolumn{4}{|c|}{ Actualización discontinua. Últimos tuits de febrero de 2013 y noviembre 2012} \\
\hline Museo Industrial del Ter. Manlleu (Barcelona) & 2.076 & 431 & 1.096 & $13 / 02 / 13$ \\
\hline Museo Nacional de Arte de Catalunya. Barcelona & 1.864 & 473 & 15.014 & $13 / 02 / 13$ \\
\hline Museo de la Vida Rural. L’Espluga de Francolí (Tarragona) & 2.871 & 2.315 & 2.771 & $13 / 02 / 13$ \\
\hline Vinseum. Museo de las Culturas del Vino de Catalunya. Vilafranca del Penedès (Barcelona) & 203 & 282 & 393 & $13 / 02 / 13$ \\
\hline Museo del Ferrocarril. Vilanova i la Geltrú (Barelona) & 347 & 99 & 431 & $11 / 02 / 13$ \\
\hline
\end{tabular}

Tabla V. Twitter

\begin{tabular}{|c|c|c|c|}
\hline Museo & Fecha alta & Amistades & "Me gusta" \\
\hline Biblioteca Museo Víctor Balaguer. Vilanova i la Geltrú (Barcelona) & $14 / 10 / 2009$ & No informa & 1.118 \\
\hline Museo de Arte Jaume Morera. Lleida & Febrero 2010 & No informa & 1.156 \\
\hline Museo de Arte Moderno de Tarragona & Enero 2011 & No informa & 494 \\
\hline Museo de Historia Sant Feliu de Guíxols (Girona) & \multicolumn{3}{|c|}{ Problemas de acceso. En realidad, es de la Asociación de Amigos, no del museo } \\
\hline Museo de Badalona. Badalona (Barcelona) & \multicolumn{3}{|c|}{ Error en el enlace: Desde Facebook se accede, indica que han cambiado url } \\
\hline Thermalia. Museo de Caldes de Montbui (Barcelona). & $10 / 08 / 2010$ & 973 & No informa \\
\hline Museo Comarcal de Cervera (Lleida) & $13 / 11 / 2009$ & 2.575 & No informa \\
\hline Museo de Gavà (Barcelona) (No propio, pertenece a Arqueoxarxa) & $24 / 05 / 2011$ & No informa & 500 \\
\hline Museo de l'Empordà. Figueres (Girona) & $04 / 01 / 2010$ & No informa & 1.267 \\
\hline Museo de Montserrat. Monistrol de Montserrat (Barcelona) & 06/10/2009 & 5.124 & No informa \\
\hline Museo del Cine. Girona & $31 / 01 / 2011$ & No informa & 1.138 \\
\hline Museo del Juguete de Catalunya. Figueres (Girona) & $16 / 09 / 2011$ & No informa & 381 \\
\hline Museo de la Ciencia y la Técnica de Catalunya. Terrassa (Barcelona) & $12 / 12 / 2008$ & No informa & 1.648 \\
\hline Museo Episcopal de Vic (Barcelona) & 08/05/2012 & 52 & No informa \\
\hline $\begin{array}{l}\text { Can Quintana. Museo de la Mediterránea. Torroella de Montgrí } \\
\text { (Girona) }\end{array}$ & $21 / 01 / 2009$ & No informa & 1.960 \\
\hline Museo Marítimo de Barcelona & Julio 2012 & No informa & 2.129 \\
\hline Museo Molí Paperer de Capellades (Barcelona) & 2010 & 4.991 & No informa \\
\hline Museo Industrial del Ter. Manlleu (Barcelona) & $27 / 01 / 2009$ & No informa & 2.284 \\
\hline Museo Nacional de Arte de Catalunya. Barcelona & $24 / 06 / 2009$ & No informa & 11.487 \\
\hline Ecomuseo de les Valls d'Àneu (Lleida) & $02 / 11 / 2011$ & No informa & 279 \\
\hline Museo de la Vida Rural. L’Espluga de Francolí (Tarragona) & $20 / 01 / 2009$ & No informa & 2.337 \\
\hline $\begin{array}{l}\text { Vinseum. Museo de las Culturas del Vi de Catalunya. Vilafranca del } \\
\text { Penedès (Barcelona) }\end{array}$ & $20 / 07 / 2010$ & No informa & 1.010 \\
\hline Museo Romántico Can Papiol. Vilanova i la Geltrú (Barcelona) & $27 / 09 / 2011$ & No informa & 479 \\
\hline Museo del Ferrocarril. Vilanova i la Geltrú (Barcelona) (2) & Marzo 2012 & No informa & 1.200 \\
\hline
\end{tabular}

Tabla VI. Facebook 
Finalmente analizamos Facebook (Tabla VI, en la página anterior) donde todos los museos que tienen alguna cuenta en redes sociales -24 para ser exactos-, la tienen en Facebook. Si bien es cierto que Facebook es todavía la más popular, sobre todo entre el público joven, recientemente se han oído voces críticas, entre ellas las del mismo creador, Mark Zuckerberg, que reconoce la pérdida de referencia y uso entre los jóvenes. Sea como fuere el $57 \%$ es realmente bajo si, además, tenemos presente el objetivo del proyecto y su proyección de futuro.

El primer museo que creó una cuenta en Facebook fue el de la Ciencia y la Técnica de Terrassa en diciembre de 2008; el último en incorporarse ha sido el Museo Marítimo de Barcelona en julio de 2012. Sobre la cantidad de seguidores debemos distinguir entre las llamadas "amistades" o los más actuales de la última versión del sistema, los "me gusta". Esta opción puede reflejarse en el conjunto de la institución, "me gusta" el museo tal, o sobre cualquiera de las actividades 0 entradas registradas. Algunos museos no ofrecen estos datos. Se ha observado algún error de enlace o problemas de acceso, así como una cuenta común para varios museos en red y no para cada institución, como en la red de museos de arqueología (Arqueoxarxa).

Se cumple el criterio en cuanto que el mayor número de seguidores corresponde al museo más grande, el Nacional de Arte de Catalunya, con casi 11.500; pero no para los dos siguientes: el Museo de Montserrat con algo más de 5.000 y el Molí Paperer con casi los 5.000 .

Una vez más, se demuestra que la actividad de publicar resulta clave para conseguir la atención y seguimiento de fans, utilizando el término popular de seguidores, más que el tiempo de permanencia en la red. Lo demuestra también el Museo Marítimo de Barcelona, que en solo siete meses ha superado los 2.100 seguidores.

Se observa una buena interacción en general, salvo algún caso entre la institución y sus seguidores (véase tabla $\mathrm{VI}$ ). El nivel de respuesta por parte de la institución es en general rápido.

\section{Conclusiones}

A partir de la pregunta de investigación planteada - iLos museos participantes en un proyecto de museos en línea están utilizando las herramientas básicas de comunicación en la web 2.0 y están presentes informando, actualizando e interactuando información y contenidos con los usuarios a través de las redes sociales?- es necesario valorar los resultados obtenidos.
La respuesta podría ser perfectamente dual: sí, porque un $57 \%$ de los museos participantes en el proyecto están presentes en redes sociales; y no, porque un $43 \%$ no lo están, y los que están plantean problemas: no siempre disponen de información actualizada; algunos enlaces dan error; hay algunos problemas de acceso; el mismo directorio de la Generalitat no tiene las URL actualizadas; presentan muy escasa presencia en Twitter, en blogs, etc. El proyecto funciona bien en su base, ofrecer vía internet acceso a las colecciones y fondos museísticos, sin duda de gran valor, pero debe mejorar $-\mathrm{y}$ mucho- en la implementación de herramientas básicas web 2.0 - jno digamos de última generación para dispositivos móviles! - y en la presencia y actividad en las redes sociales.

De los museos estudiados destacan tres por su participación en las redes y por la aplicación de las herramientas tecnológicas: el Museo Nacional de Arte de Catalunya, el Museo Marítimo de Barcelona y el de la Vida Rural de l'Espluga. Podemos tomar estas instituciones como ejemplo de buenas prácticas para otros centros. Ejemplos de referencia son dos líderes mundiales -el Louvre y la Tate Modern-, aventajados en la aplicación de tecnologías de última generación y en el uso estratégico de las redes sociales como medio de comunicación. No obstante, encontramos excelentes ejemplos de buenas prácticas más cercanos. Sin duda, el Museo Picasso de Barcelona, ganador en 2010 del premio Best of the Web Social Media, concedido en el congreso Museums and the web. Así mismo, una votación de internautas en la web MediaMusea.com en 2011 sobre las mejores experiencias en redes sociales y aplicaciones tecnológicas en España, señaló el Museo Thyssen Bornemisza de Madrid como el mejor; seguidos del Museo del Prado, el Picasso de Barcelona y el Museo de Arte Contemporáneo de Barcelona (MACBA).

En cualquier caso, el auténtico cambio debe producirse desde dentro de la institución, y quedar fundamentado en un plan de comunicación web -incluido en el plan general de comunicación-que esté basado en el nuevo concepto de comunicar y conversar frente a los tradicionales conceptos de comunicación (1), como confirma también, Martínez-Sanz en su artículo (2012) sobre la estrategia comunicativa digital en el museo.

\section{Notas}

(1) Sobre lo apuntado anteriormente, los resultados de la última encuesta anual (marzo 2013) de navegantes en red que realiza la Asociación para la Investigación de Medios de Comunicación (http://www.aimc.es/-Navegan 
tes-en-la-Red-.html) es abrumadora respecto al uso de las redes sociales: el $67,4 \%$ de internautas las utilizan a diario; de ellos, el 90,5\% usan Facebook, el 43,9\% Twitter, el $24,3 \%$ Linkedln y un $19 \%$ Tuenti.

(2) Tiene otro perfil con "Me gusta": 2.604.

\section{Referencias}

Arends, Max; et al. (2011). Museums on the web: interaction with visitors. // Handbook of research on technologies and cultural heritage: applications and environments. Hershey: IGI-Global, 2011.

Arroyo-Vázquez, Natalia; et al. (2009). Promoción de servicios de información en las redes sociales: cómo difundir nuestros servicios en la web social. [Library Instructional Material]. http://eprints.rclis.org/16587.

Badell, Joan-Isidre; Rovira, Cristòfol (2010). Visibilidad de las sedes web de los museos de Cataluña. // Revista Española de Documentación Científica 33:4: 531-52.

Badell, Joan-Isidre (2011). Museus de Catalunya i web 2.0: estudi de casos. http://diposit.ub.edu/dspace/handle/ 2445/15062.

Burton, Jane (2012) Innovation and Content in Museum Website. // II Encuentro Sobre Redes Sociales en Museos y Centros de Arte Contemporáneo, León, 11 febrero 2012. http://musac.es/index.php?ref=142400.

Celaya, Javier (2009). Más allá de la web tradicional: los museos en la web 2.0: El sector cultural en las redes sociales. // Dosdoce.com

Codina, Lluís (2004). Metodología de análisis y evaluación de recursos digitales en línea: Información y documentación digital, Barcelona: Institut Universitari de Lingüística Aplicada, Universitat Pompeu Fabra, 2004.

Estudio las nuevas tecnologías web 2.0 en la promoción de museos y centros de arte. (2006). Estudio elaborado por DosDoce y NV Asesores.

Estudio visibilidad de los museos en la web 2.0. (2009). Estudio elaborado por DosDoce.com y Abanlex Abogados.
Estudio de los museos en la era digital. (2013). Estudio elaborado por DosDoce.com.

Gómez Vilchez, Soledad (2012). Museos españoles y redes sociales: evaluación de preferencia y participación. // Telos: cuadernos de comunicación e innovación. 90, 7986.

II Encuentro sobre redes sociales en museos y centros de arte contemporáneo. Museo de Arte Contemporáneo de Castilla y León. León, 11 de febrero de 2012. http://mu sac.es/index. php?ref $=142400$

Kelly, Lynda (2010). How Web 2.0 is changing the nature of museum work. // Curator: the Museum Journal. 53:4, 405-10.

García Marco, Francisco Javier (2008). El Reto de difundir el patrimonio cultural en Internet: perspectivas y experiencias. // Scire: representación y organización del conocimiento. 14:2.

Martínez-Sanz, Raquel (2012). Estrategia comunicativa digital en el museo. // El Profesional de la Información. 21:4, 391-95.

Monistrol, Ricard; Codina, Lluís; Rovira, Cristòfol (2006). Sitios web de museos de Cataluña: análisis y propuesta de evaluación. // Hipertext.net. 4:1.

Museos en línea. http://museusenlinia.gencat.cat/

Quijano Pascual, Mario (2012). La Revolución de los museos y las instituciones culturales. // Telos: cuadernos de comunicación e innovación. 90, 55-60.

Rodríguez-Martínez, Ruth; Codina, Lluís; Pedraza, Rafael (2012). Indicadores para la evaluación de la calidad en cibermedios: análisis de la interación y de la adopción de la Web 2.0. // Revista Española de Documentación Científica. 35:1, 61-93.

Simon, Nina (2007). Discourse in the Blogosphere: what museums can learn from Web 2.0. // Museums \& Social Issues. 2:2, 257-74.

Enviado: 2013-03-28. Segunda versión: 2013-06-12 Aceptado: 2013-08-26.

\section{Anexo: Listas de museos estudiados y de museos sin presencia web 2.0}

\begin{tabular}{|c|c|c|}
\hline$N^{\circ}$ & Museo & Dirección web \\
\hline 1 & $\begin{array}{l}\text { Biblioteca Museo Víctor Balaguer. Vilanova i la Geltrú } \\
\text { (Barcelona) }\end{array}$ & http://www.victorbalaguer.cat/ \\
\hline 2 & Museo de Arte Jaume Morera. Lleida & http://www.paeria.cat/mmorera/ \\
\hline 3 & Museo de Arte Moderno de Tarragona & http://www.altanet.org/MAMT/ \\
\hline 4 & Museo de Historia de l'Hospitalet (Barcelona) & http://www.museul-h.cat \\
\hline 5 & Museo de Historia de Girona & http://www.ajuntament.gi/museuciutat \\
\hline 6 & Museo de Historia Sant Feliu de Guíxols (Girona) & http://www.guixols.cat/museu/index.html \\
\hline 7 & Museo de Badalona. Badalona (Barcelona) & http://www.museudebadalona.cat \\
\hline 8 & Thermalia. Museo de Caldes de Montbui (Barcelona) & http://www.thermalia.cat \\
\hline 9 & Museo Comarcal de Cervera (Lleida) & http://www.museudecervera.cat \\
\hline 10 & Museo de Gavà (Barcelona) & http://www.arqueoxarxa.cat/Museus/MUSEU-DE-GAVA \\
\hline 11 & Museo de l'Empordà. Figueres (Girona) & http://www.museuemporda.org \\
\hline 12 & Museo de la Noguera. Balaguer (Lleida) & http://www.museucn.com \\
\hline 13 & $\begin{array}{l}\text { Museo de la Piel de Igualada y Comarcal de Anoia } \\
\text { (Barcelona) }\end{array}$ & http://www.igualadaturisme.cat/institucio.php \\
\hline 14 & Museo de Montserrat. Monistrol de Montserrat (Barcelona) & http://www.museudemontserrat.com/ \\
\hline 15 & Museo Etnográfico de Ripoll (Girona) & http://www.museuderipoll.org/ \\
\hline
\end{tabular}


89

\begin{tabular}{|c|c|c|}
\hline 16 & Museo de Terrassa (Barcelona) & http://www.terrassa.org/museu \\
\hline 17 & Museo del Cine. Girona & http://www.museudelcinema.cat/cat/index.php \\
\hline 18 & Museo del Conca Dellà. Isona i Conca Dellà (Lleida) & http://www.parc-cretaci.com \\
\hline 19 & Museo del Juguete de Catalunya. Figueres (Girona) & http://www.mjc.cat/ \\
\hline 20 & $\begin{array}{l}\text { Museo de la Ciencia y la Técnica de Catalunya. Terrassa } \\
\text { (Barcelona) }\end{array}$ & http://www.mnactec.cat \\
\hline 21 & Museo Diocesano de Tarragona & http://www.arquebisbattarragona.cat \\
\hline 22 & Museo Episcopal de Vic (Barcelona) & http://www.museuepiscopalvic.com/ \\
\hline 23 & Museo del Mar de Lloret de Mar (Girona) & http://www.lloret.org/catala/Cultura/A0EDA3A35FF34773B57B0F8B904A5E71.asp \\
\hline 24 & $\begin{array}{l}\text { Can Quintana. Museo de la Mediterránea. Torroella de } \\
\text { Montgrí (Girona) }\end{array}$ & http://www.museudelamediterrania.org \\
\hline 25 & Museo Marítimo de Barcelona. Barcelona & http://www.museumaritimbarcelona.cat \\
\hline 26 & Muse Molí Paperer de Capellades (Barcelona) & http://www.mmp-capellades.net \\
\hline 27 & Museo de las Minas de Cercs (Barcelona) & http://www.mmcercs.cat \\
\hline 28 & Museo Municipal de Náutica del Masnou (Barcelona) & http://www.elmasnou.net/museu/ \\
\hline 29 & Museo Industrial del Ter. Manlleu (Barcelona) & http://www.mitmanlleu.org \\
\hline 30 & Museo Municipal de Montmeló (Barcelona) & http://www.montmelo.es/ca/Municipi/Equipaments.htm?id=6\&pl=68 \\
\hline 31 & Museo Municipal de Tossa de Mar (Barcelona) & http://www.tossademar.com/museu/home.htm \\
\hline 32 & Museo Nacional de Arte de Catalunya. Barcelona & http://www.mnac.es \\
\hline 33 & Museo dera Val d'Aran. Vilha e Mijaran (Lleida) & http://www.cultura.conselharan.org \\
\hline 34 & Ecomuseo de las Valls d’Àneu (Lleida) & http://www.ecomuseu.com \\
\hline 35 & $\begin{array}{l}\text { Museo de la Vida Rural. L'Espluga de Francolí } \\
\text { (Tarragona) }\end{array}$ & http://www.museuvidarural.com \\
\hline 36 & $\begin{array}{l}\text { Centre de Documentación y Museo Textil de Terrassa } \\
\text { (Barcelona) }\end{array}$ & http://www.cdmt.es \\
\hline 37 & $\begin{array}{l}\text { Museo Torre Balldovina. Santa Colona de Gramenet } \\
\text { (Barcelona) }\end{array}$ & http://www.gramenet.cat/index.php?id=3699\&no_cache=1 \\
\hline 38 & Museo de Valls (Tarragona) & http://www.ajvalls.org/obrepag.asp?web=museu\&car=turisme \\
\hline 39 & Museo Arqueològico Comarcal de Banyoles (Girona) & http://www.museusdebanyoles.cat \\
\hline 40 & $\begin{array}{l}\text { Vinseum. Museo de las Culturas del Vino de Catalunya. } \\
\text { Vilafranca del Penedès (Barcelona) }\end{array}$ & http://vinseum.cat \\
\hline 41 & $\begin{array}{l}\text { Museo Romántico Can Papiol. Vilanova i la Geltrú } \\
\text { (Barcelona) }\end{array}$ & http://www.vilanova.cat/html/papiol/index.html \\
\hline 42 & $\begin{array}{l}\text { Museo del Ferrocarril. Vilanova i la Geltrú } \\
\text { (Barcelona) }\end{array}$ & http://www.museudelferrocarril.org/ \\
\hline
\end{tabular}

Tabla VII. Museos participantes en el proyecto Museos en línea 


\begin{tabular}{|c|c|}
\hline Museo & Comentarios \\
\hline \multicolumn{2}{|l|}{ Museo de Historia de l'Hospitalet (Barcelona) } \\
\hline \multicolumn{2}{|l|}{ Museo de Historia de Girona } \\
\hline \multicolumn{2}{|l|}{ Museo de la Noguera. Balaguer (Lleida) } \\
\hline \multicolumn{2}{|c|}{ Museo de la Piel de Igualada y Comarcal de l'Anoia (Barcelona) } \\
\hline Museo Etnográfico de Ripoll (Girona) & Web en remodelación \\
\hline Museo de Terrassa (Barcelona) & $\begin{array}{l}\text { Sin web propia. Cuelga de la del Ayuntamiento. No dispone de cuenta } \\
\text { propia en redes sociales. }\end{array}$ \\
\hline \multicolumn{2}{|l|}{ Museo del Conca Dellà. Isona i Conca Dellà (Lleida) } \\
\hline Museo Diocesano de Tarragona & $\begin{array}{l}\text { Con web propia, pero no dispone de cuenta propia en redes sociales. } \\
\text { Depende del Arzobispado de Tarragona que sí tiene, incluso un blog. }\end{array}$ \\
\hline Museo del Mar de Lloret de Mar (Girona) & $\begin{array}{l}\text { Sin web propia, cuelga de la del Ayuntamiento. No dispone de cuenta } \\
\text { propia en redes sociales. }\end{array}$ \\
\hline \multicolumn{2}{|l|}{ Museo de las Minas de Cercs (Barcelona) } \\
\hline Museo Municipal de Náutica del Masnou (Barcelona) & $\begin{array}{l}\text { Sin web propia, cuelga de la del Ayuntamiento. No dispone de cuenta } \\
\text { propia en redes sociales. }\end{array}$ \\
\hline Museo Municipal de Montmeló (Barcelona) & $\begin{array}{l}\text { Sin web propia, cuelga de la del Ayuntamiento. No dispone de cuenta } \\
\text { propia en redes sociales. }\end{array}$ \\
\hline \multicolumn{2}{|l|}{ Museo Municipal de Tossa de Mar (Girona) } \\
\hline Museo dera Val d'Aran. Vilha e Mijaran (Lleida) & $\begin{array}{l}\text { Sin web propia, cuelga de la del Consell. No dispone de cuenta propia en } \\
\text { redes sociales. }\end{array}$ \\
\hline \multicolumn{2}{|c|}{ Centre de Documentación y Museo Textil de Terrassa (Barcelona) } \\
\hline \multicolumn{2}{|c|}{ Museo Torre Balldovina. Santa Colona de Gramenet (Barcelona) } \\
\hline Museo de Valls (Tarragona) & $\begin{array}{l}\text { Sin web propia, cuelga de la del Ayuntamiento. No dispone de cuenta } \\
\text { propia en redes sociales. }\end{array}$ \\
\hline Museo Arqueológico Comarcal de Banyoles (Girona) & \\
\hline
\end{tabular}

Tabla VIII. Museos que no están presentes en redes sociales 